\title{
The ultraviolet flare at the center of the elliptical galaxy NGC 4278 (Research Note)
}

\author{
A. Cardullo ${ }^{1}$, E. M. Corsini ${ }^{1}$, A. Beifiori ${ }^{1}$, L. M. Buson ${ }^{2}$, E. Dalla Bontà ${ }^{1}$, L. Morelli $^{1}$, A. Pizzella ${ }^{1}$, and F. Bertola ${ }^{1}$ \\ 1 Dipartimento di Astronomia, Università di Padova, vicolo dell’Osservatorio 2, 35122 Padova, Italy \\ e-mail: andrea.cardullo@unipd.it \\ 2 INAF, Osservatorio Astronomico di Padova, vicolo dell’'Osservatorio 5, 35122 Padova, Italy
}

Received 31 July 2009 / Accepted 25 September 2009

\section{ABSTRACT}

\begin{abstract}
Context. A large fraction of otherwise normal galaxies shows a weak nuclear activity. One of the signatures of the low-luminosity active galactic nuclei (LLAGNs) is ultraviolet variability which was serendipitously discovered in the center of some low-ionization nuclear emission-line region (LINER) galaxies.

Aims. There is a pressing need to acquire better statistics about UV flaring and variability in galaxy nuclei, both in terms of the number and monitoring of targets. The Science Data Archive of the Hubble Space Telescope was queried to find all the elliptical galaxies with UV images obtained in different epochs with the Wide Field Planetary Camera 2 (WFPC2) and possibly with nuclear spectra obtained with the Space Telescope Imaging Spectrograph (STIS) in the region of the $\mathrm{H} \alpha$ emission line. These data were found only for the elliptical radiogalaxy NGC 4278.

Methods. The UV flux of the nuclear source of NGC 4278 was measured by means of aperture photometry on the WFPC2/F218W images obtained between June 1994 and January 1995. The mass of the central supermassive black hole (SBH) was estimated by measuring the broad components of the emission lines observed in the STIS/G750M spectrum and assuming that the gas is uniformly distributed in a sphere.

Results. The nucleus of NGC 4278 hosts a barely resolved but strongly variable UV source. Its UV luminosity increased by a factor of 1.6 in a period of 6 months. The amplitude and scale time of the UV flare in NGC 4278 are remarkably similar to those of the brightest UV nuclear transients which were found earlier in other LLAGNs. The mass of the SBH was found to be in the range between $7 \times 10^{7}$ and $2 \times 10^{9} M_{\odot}$. This is in agreement with previous findings based on different assumptions about the gas distribution and with the predictions based on the galaxy velocity dispersion.

Conclusions. All the LINER nuclei with available multi-epoch UV observations and a detected radio core are characterized by a UV variable source. This supports the idea that the UV variability is a relatively common phenomenon in galaxy centers, perhaps providing the missing link between LINERs and true AGN activity.
\end{abstract}

Key words. galaxies: active - galaxies: elliptical and lenticular, cD - galaxies: individual: NGC 4278 - galaxies: nuclei ultraviolet: galaxies - black hole physics

\section{Introduction}

A large fraction of otherwise normal galaxies shows weak nuclear activity. These low-luminosity active galactic nuclei (LLAGNs) occupy the faintest end of the luminosity function of the AGNs and have very low accretion rates or radiative efficiencies onto the central supermassive black hole ( $\mathrm{SBH}$, see Ho 2008 , for a review).

One of the signatures of LLAGNs is ultraviolet (UV) variability as observed with Hubble Space Telescope (HST) in the nuclei of some low-ionization nuclear emission-line region (LINER) galaxies. There have been a number of reports after the serendipitous discovery of a UV flare in the center of the elliptical galaxy NGC 4552 (Renzini et al. 1995; Cappellari et al. 1999). The images of its nucleus obtained in a five year period with the Faint Object Camera (FOC) showed an increase of the UV luminosity by a factor of 4.5 followed by a dimming of a factor of 2. O'Connell et al. (2005) unveiled a similar phenomenon in the giant elliptical NGC 1399 using the Space Telescope Imaging Spectrograph (STIS), while a rapidly fading UV source was detected in the Virgo cluster spiral NGC 4579 (Maoz et al. 1995; Barth et al. 1996). This kind of research took on a more systematic approach by Maoz et al. (2005), who monitored the UV variability of a sample of 17 LINER galaxies with compact nuclear UV sources by means of the HST Advanced Camera for Surveys (ACS). They detected a significant UV variability in almost all the sample galaxies, which were mostly spirals, with amplitudes ranging from a few to 50 percent.

This suggests that the UV variability is a relatively common phenomenon in galaxy centers. Given this, there is a need to acquire better statistics, both in terms of the number of targets and monitoring the UV-variable nuclei. Maoz et al. (1995) already queried the HST Science Data Archive for ACS data and observed mostly spiral galaxies. We searched the Wide Field Planetary Camera 2 (WFPC2) archive for all the RC3 (de Vaucouleurs et al. 1991) elliptical galaxies having UV images obtained with the same filter in different epochs before 2008. We found 37 objects (12 LINERs, 2 Seyferts, 1 transition and $1 \mathrm{H}$ II nucleus, 17 quiescent galaxies, and 4 objects with unknown nuclear activity; Ho et al. 1997, and NASA/IPAC Extragalactic Database) with at least one F218W or F300W image. NGC 4278 was the only galaxy with multiepoch WFPC2/F218W images, which was not studied before. A nuclear spectrum obtained within a subarcsecond aperture by 
STIS in the H $\alpha$ region was also available in the HST Science Data Archive. In this paper, we present and discuss the results about the UV variability of its nucleus including an estimate of the mass of its SBH based on STIS spectroscopy.

\section{NGC 4278}

NGC 4278 is a large $(4 ! 1 \times 3 ! 8, \mathrm{RC} 3)$ and bright $\left(B_{\mathrm{T}}=11.09\right.$, RC3) elliptical galaxy. It is classified E1-2 and hosts a LINER nucleus (L1.9, Ho et al. 1997). Its total absolute magnitude is $M_{B_{\mathrm{T}}}^{0}=-20.03$ (RC3), adopting a distance of 16.1 Mpc (Tonry et al. 2001). NGC 4278 is member of the Coma I cloud (Forbes 1996). It was one of the first elliptical galaxies in which neutral hydrogen was detected (Gallagher et al. 1977) and used to infer the dark mater content at large radii (Bertola et al. 1993). It is distributed in an inclined outer ring (Raimond et al. 1981), which is possibly associated with the inner disk of ionized gas (Goudfrooij et al. 1994; Sarzi et al. 2006). The northwest side of the galaxy is heavily obscured by large-scale dust patches and filaments which seem to spiral down into the nucleus (Carollo et al. 1997).

The optical and radio properties of the nucleus have been investigated in detail (see Giroletti et al. 2005, and references therein). The radio data reveal two symmetric steep-spectrum jets on a sub parsec scale. They emerge from a flat-spectrum core and are responsible for the bulk of the emission at radio to optical frequencies in a similar way to that seen in more powerful radio loud AGNs. However, the total radio luminosity of NGC 4278 is at least 2 orders of magnitude less than those objects (Condon et al 1998). This makes NGC 4278 an LLAGN (Giroletti et al. 2005).

\section{Observations, data reduction, and analysis}

\subsection{Nuclear ultraviolet variability}

The multi-epoch images obtained with the WFPC2 and the F218W filter were retrieved from the HST Science Data Archive. A 1800 s exposure was taken on 2 June 1994 (Prog. Id. 5380, P.I. Koratkar). Two exposures of $2200 \mathrm{~s}$ and $2300 \mathrm{~s}$ were obtained on 14 January 1995 (Prog. Id. 5381, P.I. Koratkar). The exposures were taken with the telescope guiding in fine lock mode, which typically gave an rms tracking error of 0. '003. We focused our attention on the Planetary Camera (PC) chip where the nucleus of the galaxy was centered. This consists of $800 \times 800$ pixels of $0 . ' 0455 \times 0 .{ }^{\prime} 0455$ each, yielding a field of view of about $36^{\prime \prime} \times 36^{\prime \prime}$.

The images were calibrated using the standard WFPC2 reduction pipeline maintained by the Space Telescope Science Institute (STScI). Reduction steps including bias subtraction, dark current subtraction, and flat-fielding are described in detail in the WFPC2 instrument and data handbooks (Holtzman et al. 1995b; Baggett et al. 2002; McMaster et al. 2008). Subsequent reduction was completed using standard tasks in the STSDAS package of $\operatorname{IRAF}^{1}$. The bad pixels were corrected by means of a linear one-dimensional interpolation using the data quality files and the WFIXUP task. The two 1995 images were aligned and combined using IMSHIFT and knowledge of the offset shifts. Cosmic ray events and residual hot pixels were removed using the LACOS_IMA procedure (van Dokkum 2001). The cosmic

\footnotetext{
1 Imaging Reduction and Analysis Facilities is distributed by National Optical Astronomy Observatories (NOAO).
}

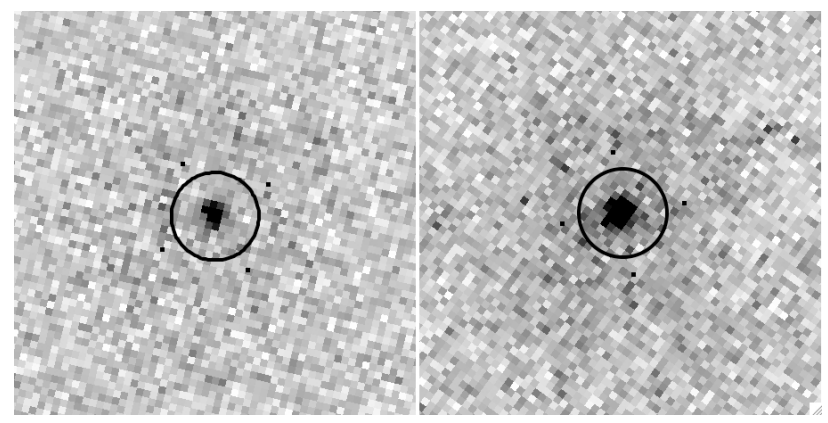

Fig. 1. WFPC2/F218W images of the NGC 4278 nucleus of 2 June 1994 (left) and 14 January 1995 (right), plotted with the same grayscale. Each panel is 2.5 on a side, north is up and east at the left. The UV flux was measured within the aperture marked by the circle $(r=0.28)$.

ray removal and bad pixel correction were checked by inspection of the residual image between the cleaned and the original frame to ensure that the nuclear region was not affected by light loss. The residual cosmic rays and bad pixels in the PC were corrected by manually editing the resulting image with IMEDIT. The sky level $\left(\sim 1\right.$ count $\left.^{-1 x e e^{-1}}\right)$ was determined from apparently empty regions in the Wide Field chips and subtracted from the PC frame after appropriate scaling.

The flux calibration was performed by adopting the Vega magnitude system (Whitmore 1995) and by taking into account the time dependence of the UV response (Mc Master \& Whitmore 2002). The presence of contaminants within WFPC2 causes a gradual build-up of material on the cold CCD faceplate of the camera, resulting in a decrease in the UV throughput. The contaminants are evaporated by periodically heating the camera to restore the instrumental throughput to its nominal value. The contamination rate is remarkably constant during each decontamination cycle, and can be accurately modeled by a simple linear decline following the decontaminations. The observed fluxes were corrected by assuming a decline in the F218W/PC normalized count rate of $(4.78 \pm 0.28) \times 10^{-3}$ per day since decontamination. This was derived during the decontamination cycles performed between April 1994 and June 1995 (Mc Master \& Whitmore 2002), which bracket the observations of NGC 4278.

Evidence of the presence of a nuclear source was found in the two final images of NGC 4278. It is barely resolved $(F W H M=0 . ' 07)$ when compared to the WFPC2/F218W point spread function (PSF, FWHM =0.'06) derived with the TINY TIM package (Krist \& Hook 1999). The total flux of the nuclear source was estimated as the flux in the circular aperture of radius of 0.28 (Fig. 1). The background level was determined as the median of the flux within the annulus of $0.37-0.51$ centered on the source. The correction for the finite aperture radius has been performed by multiplying counts by $1.18 \pm 0.01$, based on the encircled-energy value of a point source tabulated in Holtzman et al. (1995a). The errors were calculated taking in account the Poisson and CCD readout noises, charge transfer efficiency, correction for contamination, and correction for finite aperture. The UV flux of the central source of NGC 4278 in the WFPC2/F218W passband increased from $(6.94 \pm 0.46) \times 10^{-16}$ to $(10.83 \pm 0.25) \times 10^{-16} \mathrm{erg} \mathrm{cm}^{-2} \mathrm{~s}^{-1} \AA^{-1}$ from 2 June 1994 to 14 January 1995 (Fig. 2).

\subsection{Estimate of the mass of the central black hole}

The long-slit spectrum of the nucleus of NGC 4278 obtained with STIS in the region of the [N II] $\lambda \lambda 6548,6583, \mathrm{H} \alpha$, 


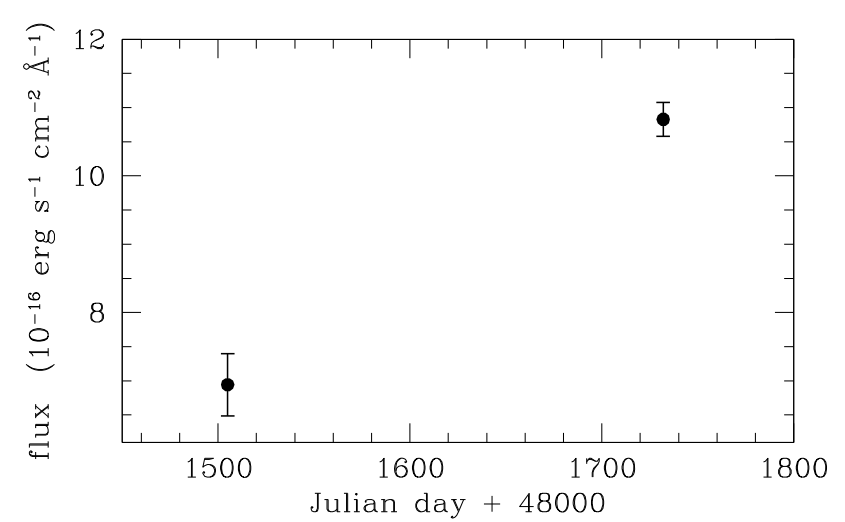

Fig. 2. UV light curve in WFPC $2 / \mathrm{F} 218 \mathrm{~W}$ band for the nucleus of NGC 4278. Points correspond to fluxes measured on 2 June 1994 and 14 January 1995, respectively.

and [S II] $\lambda \lambda 6716,6731$ emission lines (Prog. Id. 7403, P.I. Filippenko) was retrieved from the HST Science Data Archive. The G750M grating was used at the secondary tilt $\lambda_{\mathrm{c}}=6581 \AA$ covering the wavelength range $6295-6867 \AA$. The spectrum was taken with the $0.2 \times 52^{\prime \prime}$ slit placed across the galaxy nucleus with a position angle of $87^{\circ} .9$. The total exposure time was $3128 \mathrm{~s}$. The dispersion is $0.55 \AA \mathrm{pixel}^{-1}$. The instrumental resolution is $1.6 \AA(F W H M)$ corresponding to $\sigma_{\text {inst }} \approx 32 \mathrm{~km} \mathrm{~s}^{-1}$ at $\mathrm{H} \alpha$. The spatial scale of the $1024 \times 1024$ SITe CCD is 0. . $^{\prime} 05$ pixel $^{-1}$.

The spectrum was reduced using IRAF and the STIS reduction pipeline maintained by the STScI. The basic reduction steps including overscan subtraction, bias subtraction, dark subtraction, and flatfield correction are described in detail in the STIS instrument and data handbooks (Kim Quijano et al. 2007; Dressel et al. 2007). The cosmic ray events and hot pixels were removed using the task LACOS_SPEC by van Dokkum (2001). The residual bad pixels were corrected by means of a linear onedimensional interpolation using the data quality files. The wavelength and flux calibration as well as geometric correction for two-dimensional distortion were performed following the standard STIS reduction pipeline and applying the X2D task. This task corrected the wavelength scale to the heliocentric frame as well.

The central wavelengths, $F W H M$ s, and intensities of the all the observed emission lines were measured following Beifiori et al. (2009). The broad and narrow components of the emission lines were fitted with multiple Gaussians, while describing the stellar continuum with a low-order polynomial. A flux ratio of 1:2.96 was assumed for the [N II] doublet, as dictated by atomic physics (e.g., Osterbrock 1989) and both the [N II] and [S II] doublets were assumed to share a common line centroid and width. A broad component was needed to describe the $\mathrm{H} \alpha$ emission out to $0{ }^{\prime} .20$ from the center. The forbidden [N II] and [S II] lines required a broad component from -0 .' $^{\prime} 05$ to +0 .' $^{\prime} 1$. Figure 3 shows the continuum-subtracted nuclear spectrum of NGC 4278 with the fitted emission lines. It was extracted from the three central rows of the $\mathrm{H} \alpha$ STIS/G750M spectrum centered on the continuum peak. It thus consists of the central emission convolved with the STIS spatial PSF and sampled over a nearly square aperture of $0.15 \times 0 . ' 2$ (corresponding to $12 \times 16 \mathrm{pc}^{2}$ ).

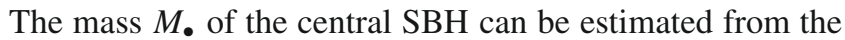
virial theorem by assuming that the gas is uniformly distributed within a sphere of radius $R$ and moves around the SBH with a
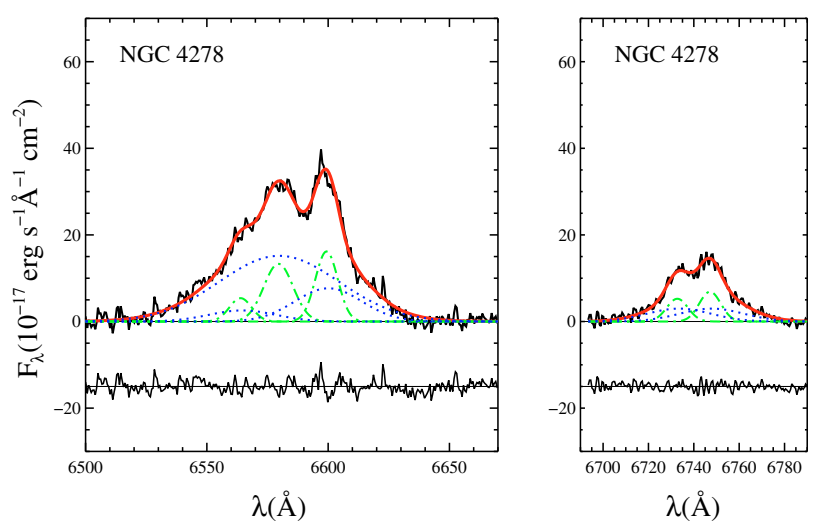

Fig. 3. Continuum-subtracted central spectrum of NGC 4278 in the $\mathrm{H} \alpha+[\mathrm{N} \mathrm{II}]$ (left panel) and [S II] (right panel) regions. In each panel, the red solid line shows the overall line blend, whereas the green dasheddotted lines and blue dotted lines show the adopted narrow and broad Gaussian components, respectively. The fit residuals also are shown, offset for better visibility.

mean velocity measured from the width of the broad component of the $\mathrm{H} \alpha$ line.

The lower limit on the size of the broad-line emitting region is given by the upper limit to the gas density as set by the forbidden lines and $\mathrm{H} \alpha$ luminosity. Following Osterbrock (1989)

$R_{\min }=\left(\frac{3 L_{\mathrm{H} \alpha}}{4 \pi f n_{\mathrm{e}}^{2} \alpha_{\mathrm{H} \alpha}^{\mathrm{eff}} h \nu_{\mathrm{H} \alpha}}\right)^{\frac{1}{3}}$

where $L_{\mathrm{H} \alpha}$ is the total luminosity of the $\mathrm{H} \alpha$ broad component, $f$ is the volume filling factor, $n_{\mathrm{e}}$ is the electron density of the gas, $\alpha_{\mathrm{H} \alpha}^{\mathrm{eff}}$ is the recombination coefficient for $\mathrm{H} \alpha, h$ is the Planck constant, and $v_{\mathrm{H} \alpha}$ is the frequency of the $\mathrm{H} \alpha$ line. The ratio of the [S II] lines sets $n_{\mathrm{e}} \approx 10^{5} \mathrm{~cm}^{-3}$, whereas the spherical and uniform distribution of the gas gives a filling factor equal to 1 . It is $L_{\mathrm{H} \alpha}=4.13 \times 10^{38} \mathrm{erg} \mathrm{s}^{-1}$ from the measured flux $(1.34 \pm 0.10) \times$ $10^{-14} \mathrm{erg} \mathrm{s}^{-1} \mathrm{~cm}^{-2}$ and assumed distance. We then find $R_{\min }=$ $0.1 \mathrm{pc}$. In this region $\sigma_{V}=1224 \pm 40 \mathrm{~km} \mathrm{~s}^{-1}$, which corresponds to a lower limit on the SBH mass of $M_{\bullet} \geq R_{\min } \sigma_{V}^{2} / G \simeq 7 \times$ $10^{7} M_{\odot}$.

The upper limit on the size of the broad-line emitting region $R_{\max }$ can be estimated from the intrinsic emissivity distribution of the gaseous sphere. An intrinsically Gaussian flux profile centered on the stellar nucleus was assumed. It has a $F W H M=0.19(15 \mathrm{pc})$ when accounting for the STIS PSF $(F W H M=0.09)$. The choice of a Gaussian parametrization is also conservative, since cuspier functions would have led us to estimate smaller $M_{\text {. }}$. We find $R_{\max }=5.9 \mathrm{pc}$ and $\sigma_{V}=$ $1127 \pm 30 \mathrm{~km} \mathrm{~s}^{-1}$ which translates into an upper limit on the $\mathrm{SBH}$ mass of $M_{\bullet} \leq R_{\max } \sigma_{V}^{2} / G \simeq 2 \times 10^{9} M_{\odot}$.

\section{Conclusions}

UV variability with amplitudes ranging from a few to 50 percent over a timescale of a decade was detected in most of the LINER nuclei observed more than once (Maoz et al. 2005) suggesting a possible link between UV flares and SBH-related activity in LLAGNs. To acquire better statistics, both in terms of the number of targets and their monitoring, we queried the HST Science Archive for all the elliptical galaxies with available UV images obtained with the WFPC2 in different epochs. 
Multi-epoch images were found only for NGC 4278, a nearby radiogalaxy known to host a LLAGN (Giroletti et al. 2005). It is characterized by a barely resolved nuclear source, which increased its UV luminosity by a factor of 1.6 in a period of 6 months. The amplitude and scale time of the variation are similar to those of the UV-brightest nuclear transients, which were earlier discovered in NGC 4552 (Renzini et al. 1995; Cappellari et al. 1999), NGC 4579 (Maoz et al. 1995; Barth et al. 1996), and NGC 1399 (O'Connell et al. 2005).

These serendipituous findings support the idea that the UV variability is a common event at the center of galaxies where SBHs reside. Some alternatives to the AGN interpretation were explored to explain the UV variability. Maoz et al. (2005) pointed out that individual supergiants in galactic nuclei are not plausible candidates to produce the observed UV flux variations. On the other hand, the even brighter Wolf-Rayet and luminous blue variable stars could only explain the variations measured in the nuclei of lower UV luminosity. The fallback of debris onto the $\mathrm{SBH}$, and collisions between precessing debris orbits (Rees 1988; Kim et al. 1999) are expected to produce bright UV/X-rays flares (Ulmer 1999). But given their rarity $\left(\approx 10^{-4} \mathrm{yr}^{-1}\right.$ per galaxy, Magorrian \& Tremaine 1999; Wang \& Merritt 2004), the stellar tidal disruptions can emerge only in all-sky deep X-ray (Donley et al. 2002; Esquej et al. 2007) and UV surveys (Gezari et al. 2006, 2008, 2009) and cannot account all the observed variable nuclei. This is particularly true for the galaxies with repeated episodes of UV variability, like NGC 4552 (Renzini et al. 1995; Cappellari et al. 1999; Maoz et al. 2005).

It was possible to estimate the mass of the SBH at the center of NGC 4278. The central width and radial extension of the broad components of the emission lines were measured over a subarcsecond aperture in the available STIS spectrum of the nucleus. If the gas is uniformly distributed within a sphere then it is $7 \times 10^{7} \leq M_{\bullet} \leq 2 \times 10^{9} M_{\odot}$. This is consistent with the predictions of the $M_{\bullet}-\sigma_{c}$ relations by Ferrarese \& Ford (2005) and Lauer et al. (2007) when adopting a central velocity dispersion $\sigma_{\mathrm{c}}=333 \pm 8 \mathrm{~km} \mathrm{~s}^{-1}$ (Beifiori et al. 2009). There is also a good agreement with the upper limits on $M$. given by Beifiori et al. (2009). They measured the nuclear width of the narrow component of the [N II] $\lambda 6583$ emission line and modelled it as due to the Keplerian motion of a thin disk of ionized gas around the putative SBH. They found $M_{\bullet} \leq 5 \times 10^{7}$ for a nearly edge-on disk and $M_{\bullet} \leq 2 \times 10^{8} M_{\odot}$ for a nearly face-on disk.

According to Giroletti et al. (2005) this SBH is active and able to produce the relativistic jet, responsible for most of the emission at optical and radio frequencies of this LLAGN. It is the same process as the ordinary radio loud AGNs despite a much lower power. The AGN interpretation is a promising way to explain the UV variability. In fact, all the LINER nuclei with multi-epoch observations and a detected radio core are characterized by UV variable sources (Maoz et al. 2005). This is the case of NGC 4278, too, suggesting that UV variability could provide the missing link between LINERS and true AGN activity. Unfortunately, it is the only elliptical galaxy observed by HST at different epochs in the UV and which was not studied before. This fact does not allow us to derive any firm statistical conclusion about the frequency of UV flares at the center of elliptical galaxies. Nevertheless, out of 37 galaxies, the only object with multi-epoch UV observations turned out to be variable. This is a further suggestion that this phenomenon may be quite common. Additional imaging with the Wide Field Camera 3 recently installed on HST to monitor the UV variablity in a statistically significant sample of quiescent and active nuclei and STIS spectra to measure their SBHs are highly desirable to gain insights into this subject in the near future.

Acknowledgements. A.C. acknowledges the Space Telescope Science Institute for hospility while this work was in progress. We thank Massimo Stiavelli and Michele Cappellari for useful discussions and their helpful comments. This work was made possible through grants CPDA068415 and CPDA089220 by Padua University. This research has made use of the Lyon-Meudon Extragalactic Database (LEDA) and NASA/IPAC Extragalactic Database (NED).

\section{References}

Baggett, S., et al. 2002, WFPC2 Data Handbook, Version 4.0, STScI, Baltimore Barth, A. J., Reichert, G. A., Filippenko, A. V., et al. 1996, AJ, 112, 1829 Beifiori, A., Sarzi, M., Corsini, E. M., et al. 2009, ApJ, 692, 856 Bertola, F., Pizzella, A., Persic, M., \& Salucci, P. 1993, ApJ, 416, L45 Cappellari, M., Renzini, A., Greggio, L., et al. 1999, ApJ, 519, 117 Carollo, C. M., Franx, M., Illingworth, G. D., \& Forbes, D. A. 1997, ApJ, 481, 710

Condon, J. J., Cotton, W. D., Greisen, E. W., et al. 1998, AJ, 115, 1693 de Vaucouleurs, G., de Vaucouleurs, A., Corwin, H. G., et al. 1991, The Third Refecence Catalog of Bright Galaxies (New York: Springer-Verlag) (RC3) Donley, J. L., Brandt, W. N., Eracleous, M., \& Boller, T. 2002, AJ, 124, 1308 Dressel, L., et al. 2007, STIS Data Handbook, Version 5.0, STScI, Baltimore Esquej, P., Saxton, R. D., Freyberg, M. J., et al. 2007, A\&A, 462, L49 Ferrarese, L., \& Ford, H. 2005, Space Sci. Rev., 116, 523 Forbes, D. A. 1996, AJ, 112, 1409

Gallagher, J. S., Knapp, G. R., Faber, S. M., \& Balick, B. 1977, ApJ, 215, 463 Gezari, S., Martin, D. C., Milliard, B., et al. 2006, ApJ, 653, L25 Gezari, S., Basa, S., Martin, D. C., et al. 2008, ApJ, 676, 944

Gezari, S., Heckman, T., Cenko, S. B., et al. 2009, ApJ, 698, 1367 Giroletti, M., Taylor, G. B., \& Giovannini, G. 2005, ApJ, 622, 178 Goudfrooij, P., Hansen, L., Jorgensen, H. E., \& Norgaard-Nielsen, H. U. 1994, A\&AS, 105, 341

Ho, L. C. 2008, ARA\&A, 46, 475

Ho, L. C., Filippenko, A. V., \& Sargent, W. L. W. 1997, ApJS, 112, 315 Holtzman, J. A., Hester, J. J., Casertano, S., et al. 1995a, PASP, 107, 156 Holtzman, J. A., Burrows, C. J., Casertano, S., et al. 1995b, PASP, 107, 1065

Krist, J., \& Hook, R. 1999, The Tiny Tim User's Guide, STScI, Baltimore Kim, S. S., Park, M.-G., \& Lee, H. M. 1999, ApJ, 519, 647

Kim Quijano, J., et al. 2007, STIS Instrument Handbook, Version 8.0, STScI, Baltimore

Lauer, T. R., Faber, S. M., Richstone, D., et al. 2007, ApJ, 662, 808

Magorrian, J., \& Tremaine, S. 1999, MNRAS, 309, 447

Maoz, D., Filippenko, A. V., Ho, L. C., et al. 1995, ApJ, 440, 91

Maoz, D., Nagar, N. M., Falcke, H., \& Wilson, A. S. 2005, ApJ, 625, 699 589, L21

Mc Master, M., \& Whitmore, B. 2002, WFPC2 Instruments Science Report, 02-07, STScI, Baltimore

McMaster, Biretta, et al. 2008, WFPC2 Instrument Handbook, Version 10.0, STScI, Baltimore

O’Connell, R. W., Martin, J. R., Crane, J. D., et al. 2005, ApJ, 635, 305

Osterbrock, D. E. 1989, Astrophysics of Gaseous Nebulae and Active Galactic Nuclei (Mill Valley: Univ. Science Books)

Raimond, E., Faber, S. M., Gallagher, J. S., III, \& Knapp, G. R. 1981, ApJ, 246, 708

Rees, M. J. 1988, Nature, 333, 523

Renzini, A., Greggio, L., di Serego-Alighieri, S., et al. 1995, Nature, 378, 39 Sarzi, M., Falcón-Barroso, J., Davies, R. L., et al. 2006, MNRAS, 366, 1151 Tonry, J. L., Dressler, A., Blakeslee, J. P., et al. 2001, ApJ, 546, 681

Ulmer, A. 1999, ApJ, 514, 180

van Dokkum, P. G. 2001, PASP, 113, 1420

Wang, J., \& Merritt, D. 2004, ApJ, 600, 149

Whitmore, B. 1995, in Calibrating Hubble Space Telescope: Post Servicing Mission, ed. A. Koratkar, \& C. Leitherer, STScI, Baltimore, 269 